\title{
The Study of Acute Aquatic Ecological Threshold of Silver Nanoparticles Based on the Acute Toxicity of Aquatic Species
}

\author{
Longchao $\mathrm{He}^{1, \mathrm{a}}$, Tongzhou Liu ${ }^{1, \mathrm{~b}}$ \\ ${ }^{1} 1$ Harbin Institute of Technology ShenZhen Graduate School, ShenZhen Key Laboratory of Water \\ Resource Utilization and Environmental Pollution Control, ShenZhen 518055, China.
}

${ }^{a}$ E-mail: 18792452577@163.com

bCorresponding author: liutongzhou@hitsz.edu.cn

\begin{abstract}
Keywords: silver nanoparticles (AgNPs), ecological threshold, species sensitivity distributions (SSDs), predicted no effect concentration (PNEC).

Abstract. Silver nanoparticles (AgNPs), as a new material with good properties, has been widely used in many fields. With the increasing production and usage of it, more and more AgNPs are entering the water environment, which was suspected to impose ecological risk to the aquatic ecosystem. Based on the acute aquatic toxicity data of AgNPs, this paper established the species sensitivity distributions (SSDs) curve of silver nanoparticles in water environment. Referring to the proposed method of establishing PNEC in Europe TGD, acute aquatic ecological threshold of AgNPs was calculated, and it is $7.35 \mu \mathrm{g} / \mathrm{L}$. This work attempts to provide a reference for the environmental impact assessment of silver nanoparticles. The results showed that current concentration of AgNPs in the water environment would have no significant toxic effects on aquatic organisms. However, the environmental impact of AgNPs still needs to pay more attention.
\end{abstract}

\section{Introduction}

Nanoparticles (NPs) are defined as materials with two or three-dimensional scale between 1 100 nm, which have surface effect, quantum size effect, small size effect and macroscopic quantum tunneling effect. Due to the excellent physicochemical and antimicrobial properties, silver nanoparticles (AgNPs) were widely used in a range of consumer goods such as clothing, household appliances, food packing, and personal care products [1]. With the widespread manufacture and use, AgNPs are inevitably released into the environmental [2]. The studies have shown that AgNPs are toxic to algae $[3,4]$, plankton [5], and fish [6,7]. Therefore, concerns have been raised about potential risk effects of AgNPs on aquatic ecological system. However, there are seldom studies have been done on the risk assessment of aquatic ecological of silver nanoparticles. The present study attempts to fill this knowledge gap by calculate the acute aquatic ecological threshold.

In ecological risk assessment, the predicted no effect concentration (PNEC) is usually used as environmental threshold. In order to deduce the PNEC, the species sensitivity distributions (SSDs) curve method is increasingly used to assess the ecological risk effects and has been accepted by lots of scholars [8]. Compared to traditional deterministic quotient approaches, SSDs have greater statistical significance and ecological meaning. SSDs are usually constructed by development cumulative probability distributions of NOEC (no observed effect concentrations) or $\mathrm{LC}_{50}$ (half lethal concentrations)/EC $\mathrm{E}_{50}$ (half effective concentration) based on various statistical methods. However, it is unfortunate that there are lack of data of silver nanoparticles' NOEC. In the present study, we use the acute toxicity data $\left(\mathrm{LC}_{50} / \mathrm{EC}_{50}\right)$ of aquatic species to deveop the SSD curve and calculate the acute aquatic ecological threshold $\left(\mathrm{PNEC}_{\text {acute }}\right)$. The purpose of this study is to provide a reference for the aquatic ecological risk assessment of silver nanoparticles.

\section{Materials and methods}

Data obtain and preparation. Because the toxicity data of AgNPs were not retrieved in the authoritative toxicity database (USEPA ECOTOXIC), we have consulted a large number of researches on the biological toxicity of AgNPs at home and abroad, and obtained many $\mathrm{LC}_{50} / \mathrm{EC}_{50}$ of aquatic species. In the selection of acute toxicity data, the principia of accuracy, appropriateness and 
reliability are followed to ensure the quality of data [9]. In order to ensure that the toxicity data have ability to represent the aquatic ecosystems, toxicity data of biological species should cover three trophic levels of algae, crustaceans and fish, and the number of species should be greater than eight. At the same conditions (experimental temperature, barometric, experimental water type and exposure time, etc.) of the same species were used to obtain the minimum value when there are more than one data, and the different data for the same species were used to obtain the geometric averages [10]. After being prepared by filter, the toxicity data used as establish SSDs curves are shown in table 1.

Table 1 Acute toxicity of aquatic species of AgNPs

\begin{tabular}{cccc}
\hline Species & $\begin{array}{c}\text { Particle Size of } \\
\text { AgNPs }[\mathrm{nm}]\end{array}$ & $\begin{array}{c}\mathrm{LC}_{50} / \mathrm{EC}_{50} \\
{[\mu \mathrm{g} / \mathrm{L}]}\end{array}$ & $\begin{array}{c}\text { Data } \\
\text { Sources }\end{array}$ \\
\hline M. aeruginosa & $20-50$ & 1000 & {$[3]$} \\
S. bibraianum & $26.6 \pm 8.8$ & 190 & {$[4]$} \\
C. vulgaris & $84.6 \pm 14.38$ & 890 & {$[11]$} \\
C. reinhardtii & $25 \pm 13$ & 87 & {$[12]$} \\
O. latipes & $20-30$ & 40 & {$[5]$} \\
C. quadrangula & $20-30$ & 67 & {$[5]$} \\
D.rerio & $20-30$ & 7070 & {$[5]$} \\
O. mykiss & $10-35$ & 100 & {$[6]$} \\
O. latipes & $1-20$ & 4570 & {$[7]$} \\
L. japonicus & 81 & 300 & {$[13]$} \\
\hline
\end{tabular}

Establishment of SSDs. In this study, the SSDs curve was established according to the following procedure: Firstly, arranging the toxicity data in ascending order and numbering; Secondly, the logarithm of the toxicity data as the abscissa, dividing the numbering of each data by the total number of data plus one as the ordinate, plotting the scatter plot; Thirdly, using the Origin 9.0 to fit the toxicity data points for distribution curve, and determining the optimal SSD curve by the correlation coefficient. At the present study, Log-Normal and Logistic distributions are used to fit the acute aquatic toxicity data of AgNPs.

Calculate PNEC $_{\text {acute }}$. In this paper, the SSDs is used to calculate the threshold of acute aquatic ecological $\left(\mathrm{PNEC}_{\text {acute }}\right)$. For the calculation of the $\mathrm{PNEC}_{\text {acute }}$ under short-term high-dose exposure, we mainly draw on the calculation method of Europe TGD [14]. Under 95\% confidence, the SSD curve was used to estimate the concentration of AgNPs that the 5\% species was affected, we define it as the

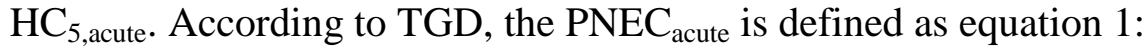

$\mathrm{PNEC}_{\text {acute }}=\mathrm{HC}_{5, \text { acute }} / \mathrm{AF}$.

In the Eq. 1, AF is an assessment factor between 5 and 1, which reflects the uncertainty in evaluation. Since the toxicity data is not sufficient, we used the conservative AF to calculate $\mathrm{PNEC}_{\text {acute, }}$, and took it as 5.

\section{Results and discussion}

SSDs of AgNPs. There are ten valid acute toxicity data of aquatic species to establish the SSDs curve. Fig 1 and Fig 2 shows the SSDs curves fitting by Log-Normal and Logistic distributions under 95\% confidence. The abscissa is the logarithm of the toxicity data, and the ordinate is the proportion of the affected species in the aquatic ecosystem. Fig 1 is the Logistic fitting curve, the P value of its F-test is $1.14 \times 10^{-7}$, and the correlation coefficient $\mathrm{R}^{2}$ is 0.99 . Fig 2 is the Log-Normal fitting curve, the $\mathrm{P}$ value of its F-test is $1.15 \times 10^{-7}$, and the correlation coefficients $\mathrm{R}^{2}$ is also 0.99 . The F-test of the two fitting curves are both less than 0.05 and the correlation coefficients are both high, which indicate that the two fitting curves are related to the toxicity points at $95 \%$ confidence level. Therefore, we can assume that both curves can be used to fit the SSDs of AgNPs, and they all have very excellent results. 


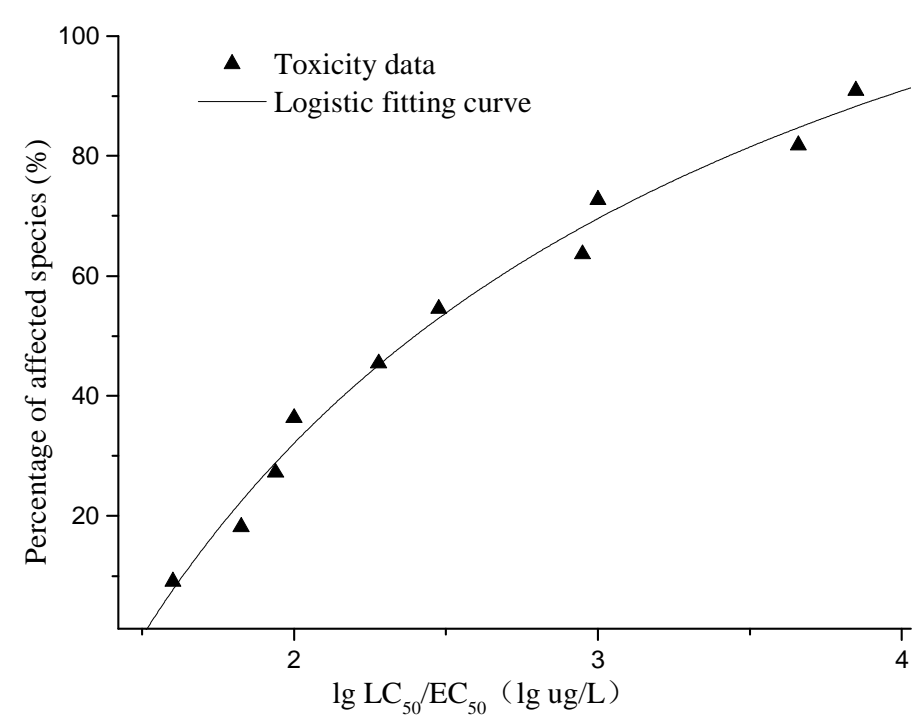

Figure 1 The SSDs curve fitting by Logistic

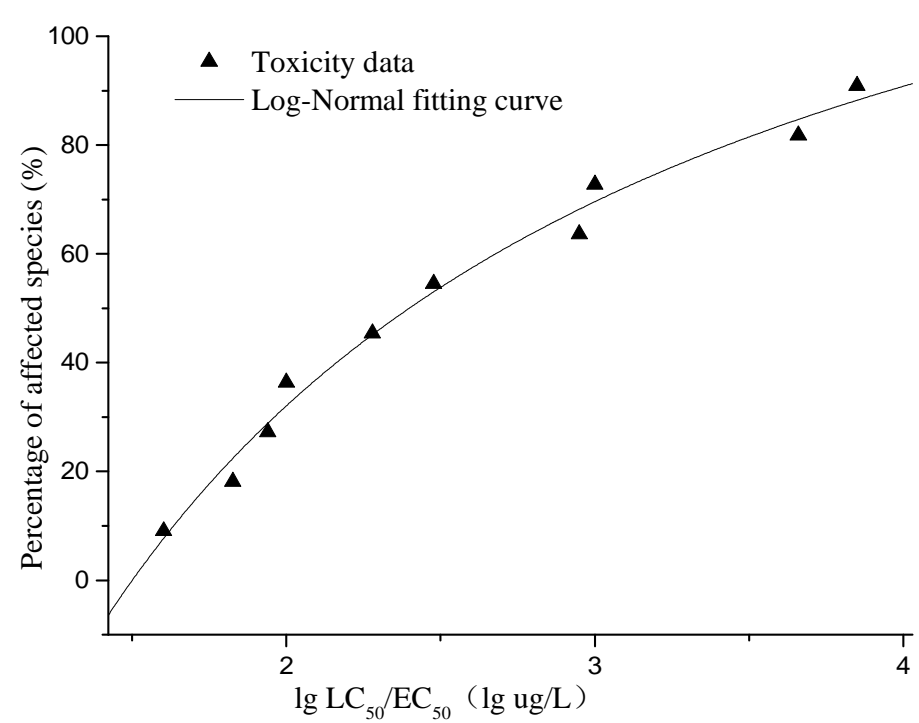

Figure 2 The SSDs curve fitting by Log-Normal

Results of PNEC $\mathbf{P a t e}_{\text {acu }}$. As mentioned earlier, we used the SSDs curve to compute $\mathrm{HC}_{5 \text {,acute. }}$. Since the two curves have both very good fitting results, we have calculated the $\mathrm{HC}_{5 \text {,acute }}$ of the two curves separately. The results showes that $\mathrm{HC}_{5 \text {,acute }}$ of the Logistic and Log-Normal curves are $36.75 \mu \mathrm{g} / \mathrm{L}$ and $36.72 \mu \mathrm{g} / \mathrm{L}$, respectively. Obviously, there is no significant difference between the two calculations. Thus, we use the arithmetic mean of the two results as the $\mathrm{HC}_{5 \text {,acute value for the next }}$ step calculation, and it is $36.74 \mu \mathrm{g} / \mathrm{L}$. According to Eq. 1, we can calculate the $\mathrm{PNEC}_{\text {acute }}$ of $\mathrm{AgNPs}$ is $7.35 \mu \mathrm{g} / \mathrm{L}$. Because of the conservative assessment factor value, the acute aquatic thresholds of the silver nanoparticles we get are relatively safe.

Environmental impact assessment. In fact, there is little research on the concentration of silver nanoparticles in the environment. The study of Gottschalk et al [15] showed that the predicted concentrations of AgNPs in surface water in the United States and Europe were 0.09-0.43 ng/L and 0.59-2.16 ng/L, respectively. Mitrano et al [16] detected the concentration of AgNPs in the effluent was between 100-200 ng/L. These studies indicate that the concentration of AgNPs in water is at a low level, and compared to the acute aquatic ecological threshold found in this paper, we can assume that the current concentration of AgNPs in the water environment would not produce significant toxic effects on aquatic organisms. 


\section{Conclusions}

At this study, based on the acute data of ten representative aquatic organisms, the SSDs curve of AgNPs in the water environment was established by two distribution curves fitting, and the acute aquatic ecological threshold $\left(\mathrm{PNEC}_{\text {acute }}\right)$ of AgNPs was $7.35 \mu \mathrm{g} / \mathrm{L}$. The results show that the concentration of silver nanoparticles in water environment is not enough to produce obvious toxic effects on aquatic organisms and aquatic ecosystems. However, with silver nanoparticles applied more and more widely, its content in water environment is also increasing, and it is necessary for people to pay more attention to the impact of silver nanoparticles on aquatic organisms and ecosystems.

\section{Acknowledgements}

Acknowledgement is owed to the financial support from the Shenzhen Innovative Technology Fund (Grant No. JSGG20170413152540284).

\section{References}

[1] B. Nowack, H.F. Krug, M. Height: Environ. Sci. Technol. 45 (4) (2011), p. 1177-1183.

[2] B. Nowack: Science. 330 (2010), p. 1054-1055.

[3] Dl. Jiang, L. Yue, D. Ma, et al: Environ. Chem. 31(3) (2012), p. 296-301.

[4] M.H. Park, K.H. Kim, H.H. Lee, et al: Biotechnol. Lett. 32(3) (2010), p. 423-428.

[5] R.J. Griffitt, J. Luo, J. Gao, et al: Environ. Toxicol. Chem. 27 (2008), p. 1972-1978.

[6] T.M. Scown, E.M. Santos, B.D. Johnston, et al: Toxicol. Sci. 115(2) (2010), p. 521-524.

[7] Y. Wu: University of Science and Technology of China. 2010.

[8] C.L.Feng, F.C. Wu, Y.S. Mu, et al: Environ. Sci. Technol. 47 (2013), p. 11382-11383.

[9] B.L. Lei, S.B. Huang, Z.J. Wang: Progress in Chemistry. 21(2/3) (2009), p. 350-358.

[10] R.J. Griffitt, J. Luo, J. Gao, et al: Environ. Toxicol. Chem. 27(9) (2010), p. 1972-1978.

[11]M. Yooiam, R. Chaichana, T. Satapanajaru: Chem. Spec. Bioavail. 26(4) (2014), p. 257-265.

[12]E. Navarro, F. Piccapietra, B. Wagner, et al: Environ. Sci. Technol. 42(23) (2008), p. 8959-8964.

[13] K. Bilberg, H. Malte, T. Wang, et al: Aquat. Toxicol. 96(2) (2010) , p. 159-165.

[14] European Commission. (2003), TGD information on http://europa.eu.int.

[15] F. Gottschalk, T. Sonderer, R.W. Scholz, et al: Environ. Sci. Technol. 43 (2009), p. 9216-9222.

[16] D.M. Mitrano, E.K. Lesher, A. Bednar, et al: Environ. Toxicol. Chem. 31(1) (2012), p. $115-121$. 